\title{
Diontos
}

Revista de Comunicación Digital

\section{Social Media Live Streaming (SMLS) en medios digitales: el uso de Periscope, Facebook Live y YouTube Live en cibermedios chilenos}

\section{Social Media Live Streaming (SMLS) in digital media: the use of Periscope, Facebook Live and YouTube Live in Chilean cybermedia}

Alexis Apablaza-Campos

alexis.apablaza@uniacc.edu

Universidad UNIACC (Chile)

\section{Alejandro Morales Vargas}

amorales@uchile.cl

Universidad de Chile

\section{Carlos Lopezosa}

carlos.lopezosa@upf.edu

Universitat Pompeu Fabra

\section{(D) Josep Salvat}

josep.salvat@uab.cat

Universitat Autònoma de Barcelona

\section{Lluís Codina}

lluis.codina@upf.edu

Universitat Pompeu Fabra

Recibido: 09/04/2019

Aceptado: 24/07/2019

\section{Resumen}

El Social Media Live Streaming (SMLS) es una nueva forma de emisión de vídeos interactivos en directo que utiliza redes sociales específicas, como Periscope, o bien funcionalidades de redes sociales generalistas, como Facebook o YouTube. El SMLS presenta un gran potencial para medios de comunicación porque su carácter interactivo, además de proporcionar datos directos de recepción, puede mejorar el engagement con sus públicos.

En este trabajo se presenta una caracterización del SMLS desde el punto de vista de su uso periodístico. Además, se presenta un estudio de caso llevado a cabo sobre el uso del SMLS por parte de medios chilenos durante la cobertura del $30^{\circ}$ aniversario del Plebiscito Nacional de 1988, considerado el punto de arranque de la recuperación de la democracia.

En concreto, se estudia el uso de Periscope/Twitter, Facebook Live y YouTube Live en los siguientes medios Teletrece, La Tercera, TVN 24 Horas, CNN Chile, Ahora Noticias, Cooperativa y Publimetro Chile. Los resultados, además de aportar datos concretos de un uso periodístico altamente significativo de este tipo de publicaciones, confirman la importancia que el SMLS puede tener en el futuro de los cibermedios como un formato adicional que otorgue mayor visibilidad a la cobertura de hechos noticiosos.

\section{Palabras clave}

Social Media Live Streaming; Facebook Live; YouTube Live; Periscope; cibermedios

Cómo citar este artículo:

Apablaza-Campos, A.; Morales Vargas, A.; Lopezosa, C.; Salvat, J.; Codina, L. (2020). Social Media Live Streaming (SMLS) en medios digitales: el uso de Periscope, Facebook Live y YouTube Live en cibermedios chilenos. Dígitos. Revista de Comunicación Digital, 6: 219-238. DOI:10.7203/rd.v1i6.145 


\section{Abstract}

Social Media Live Streaming (SMLS) is a new form of live interactive video broadcast that uses specific social networks, such as Periscope, or general social networking features, such as Facebook or YouTube. SMLS has great potential for media because its interactive nature, in addition to providing direct reception data, can improve engagement with its audiences.

In this work, a characterization of the SMLS is presented from the point of view of its journalistic use. In addition, a case study carried out on the use of the SMLS by Chilean media is presented during the coverage of the 30th anniversary of the 1988 National Plebiscite, considered the starting point for the recovery of democracy.

In particular, the use of Periscope / Twitter, Facebook Live and YouTube Live is studied in the following media: Teletrece, La Tercera, TVN 24 Horas, CNN Chile, Ahora Noticias, Cooperativa and Publimetro Chile. The results, in addition to providing concrete data of a highly significant journalistic use of this type of publications, confirm the importance that the SMLS can have in the future of the digital news media as an additional format that gives greater visibility to the coverage of news events.

\section{Keywords}

Social Media Live Streaming; Facebook Live; YouTube Live; Periscope; digital news media

\section{Social Media Live Streaming (SMLS) en medios digitales:} el uso de Periscope, Facebook Live y YouTube Live en cibermedios chilenos

\section{Introducción}

El objeto de estudio de este trabajo es el Social Media Live Streaming (en adelante, SMLS) y sus posibilidades de utilización por parte de los medios de comunicación digitales (cibermedios).

Por tanto, el objetivo de este trabajo consiste, primero, en presentar una caracterización general del SMLS con especial énfasis en su utilización periodística. En segundo lugar, en llevar a cabo un estudio de caso del SMLS para la cobertura de un suceso informativo de gran relevancia social a través del cual, sea posible comparar su uso por parte de diferentes medios.

El problema de investigación consiste en cómo caracterizar el SMLS para aplicaciones periodísticas y desarrollar un estudio de caso con el objetivo de avanzar en su comprensión ya que, como es sabido, es gracias a esta modalidad de estudio que podemos ir más allá de aportes teóricos en el ámbito de la comunicación social (Coller, 2005; Yin, 2018; Flyvbjerg, 2013; Thomas, 2011).

La pregunta de investigación abordada es: ¿de qué forma podemos plantear un estudio de caso significativo que, a su vez, permita realizar estimaciones fiables sobre para poder hacer estimaciones fiables sobre el rendimiento periodístico del SMLS?

La motivación es que los medios de comunicación digitales (o los medios de comunicación, sin más) necesitan modelos o formatos de narración que aumenten el engagement 
(entendido como implicación, participación y compromiso) con su público, como una de las formas vitales de asegurar su sostenibilidad (Fundación Gabriel García Márquez para el Nuevo Periodismo Iberoamericano, 2016; Apablaza-Campos, Codina, \& Pedraza-Jiménez, 2018a).

Los métodos utilizados para este trabajo han consistido, por un lado, en una revisión sistematizada del SMLS, tomando como batería de artículos una serie de aportaciones teóricas y profesionales sobre el tema principal, y por otro lado, en el diseño y desarrollo de un estudio de caso. Para este último, utilizamos la cobertura de medios de comunicación chilenos del $30^{\circ}$ aniversario del Plebiscito Nacional de 1988 , considerado como el hito que facilitó la posterior recuperación de la democracia en Chile y que, por tanto, presenta un notable interés periodístico. En el apartado dedicado al diseño de la investigación presentamos estos aspectos con más detalle.

\subsection{Caracterización del SMLS}

En 2016, el CEO y fundador de Facebook, Mark Zuckerberg, afirmó que "el avance más rápido de los próximos cinco años será principalmente en el vídeo" (Ingram, 2016). Tres años más tarde, hay una serie de factores que permiten sostener esta idea desde el punto de vista de la comunicación digital:

- El segundo sitio más visitado del mundo es YouTube y Netflix es el número 18 (hay otros 4 sitios de vídeo que están entre los 20 primeros, pero se trata de sitios de pornografía) (SimilarWeb, 2019).

- 'Vídeo' fue la cuarta tendencia de búsqueda más importante en Google durante 2018 (Kemp, 2019, p. 55).

- Un 92\% de los cibernautas reproduce vídeos en línea y un 58\% visualiza contenidos de televisión en streaming (Kemp, 2019, p. 59).

- En 2018, las aplicaciones móviles de vídeos fueron la segunda categoría con más descargas en iPhone y la octava en Android (Kemp, 2019, p. 183).

La industria periodística no ha estado ajena a este fenómeno, por lo cual es posible encontrar diversos estudios sobre cómo los contenidos de vídeo han cobrado cada vez más relevancia, tanto en las estrategias digitales como en las publicaciones de perfiles sociales de diferentes cibermedios en todo el mundo (Kalogeropoulos, Cherubini, \& Newman, 2016; Hansen \& Goligoski, 2018).

Las experiencias audiovisuales de tipo interactivo tienen un papel fundamental dentro de las estrategias de vídeo en las redes sociales (Argila, 2017), y una de ellas es el vídeo en directo, entendido como Social Media Live Streaming (SMLS), que cuenta con una serie de características que lo diferencian de una emisión convencional:

1. Realizar y distribuir videos en directo, en diferentes formatos, a través de las redes sociales, mediante aplicaciones propias asociadas a ellas.

2. Proporcionar al emisor una retroalimentación instantánea: número de usuarios conectados, mensajes y reacciones de sus audiencias (Apablaza-Campos \& Codina, 2018b, p. 164). 
Las tecnologías SMLS pueden clasificarse en dos tipos atendiendo a las plataformas que utilizan: (1) redes sociales de emisión en directo, como Periscope y Twitch, y (2) funcionalidades live streaming en plataformas o redes sociales generalistas, como $\mathrm{Fa}$ cebook Live, YouTube Live e Instagram Live Stories. A esta segunda clasificación se sumará pronto Linkedln Live, que actualmente está en fase beta y tiene previsto su lanzamiento a finales de 2019 (Lunden, 2019).

A efectos de clarificación, las plataformas como las mencionadas, las denominaremos canales. Por tanto, Periscope, y Facebook Live, son canales de SMLS y así nos referiremos a ellos en este trabajo.

En cuanto a su aplicación en medios de comunicación digitales, estas pueden distribuirse, al menos, en cinco tipos principales:

1) Emisión de ruedas de prensa

2) Cobertura de sucesos de última hora

3) Entre bastidores (backstage) de programas

4) Reemisión de señal televisiva

5) Experiencias de realidad virtual y/o aumentada (Morales Vargas, 2018)

Por otro lado, en relación a los canales SMLS mencionados, es importante tener presente que Twitch está enfocado en los contenidos de videojuegos, aunque se ha abierto paulatinamente a medios digitales como Buzzfeed (Southern, 2018); y que Instagram Live Stories solo permite emisiones desde teléfonos móviles y que los resultados acumulados en cada publicación solo están disponibles para el emisor del contenido (Instagram, 2019).

\subsubsection{Periscope/Twitter}

En enero de 2015, Periscope era otra empresa tecnológica emergente de Silicon Valley, cuyo objetivo era la creación de una app de live streaming. Twitter se interesó en ella y, tras comprarla por 100 millones de dólares, lanzó en marzo de ese año la red social de vídeos en directo (Panzarino \& Tsotsis, 2015). Tal como se puede ver en su sitio web, sus desarrolladores explican claramente cuál ha sido su objetivo principal desde su nacimiento:

Nos entusiasmamos con la idea de descubrir el mundo a través de ojos ajenos. ¿Y si pudieras ver a través de los ojos de un manifestante en Ucrania? ¿O mirar el amanecer desde un globo aerostático en Capadocia? Puede sonar descabellado, pero quisimos crear lo más parecido a la teletransportación. Si bien hay muchas formas de descubrir eventos y lugares, nos dimos cuenta de que no hay mejor forma de sentir un lugar en un instante determinado que mediante un video en directo. Una imagen podrá valer más que mil palabras, pero un video en directo puede transportarnos al lugar y mostrarnos lo que allí ocurre (Periscope, 2017).

En tan solo cuatro meses la app ya contaba con más de 10 millones de descargas, 2 millones de usuarios activos al día y una visualización de contenido en directo diario equivalente a 40 años (Aguayo, 2015). Así, fue reconocida como la mejor aplicación del 2015 por Apple (Eadicicco, 2015). 
Desde 2017, Periscope está totalmente integrada a su matriz Twitter, al punto que los vídeos en directo pueden emitirse en al mismo tiempo (simulcasting) por ambas plataformas. Los usuarios de la red de microblogging al realizar su primer live y aceptar las condiciones, a su vez, pueden crear de forma automática un perfil en la red social de tipo SMLS con la correspondiente notificación a sus contactos de esta emisión (Dotan, 2017).

En todo caso, la primera cobertura periodística publicada a través de un vídeo en directo a través de las redes sociales fue realizada por Paul Levis, de The Guardian, el 28 de abril de 2015 en la cobertura de la manifestación de afroamericanos contra la violencia policial en la ciudad estadounidense de Baltimore, Maryland (Levis, 2015).

Meses más tarde, Paul Ronzheimer, de Bild, fue enviado a la isla griega de Kos para seguir la travesía de los refugiados sirios realizando emisiones SMLS con una narración de la historia como si fuese un indocumentado más. Luego explicó en una entrevista que "en Alemania hay discusiones grandes sobre la intensidad de la cobertura periodística de la historia. Pero en Periscope todos pueden verlo en vivo... Pude grabar sin ser tan obvio que era parte de un medio" (Pichihua, 2015).

Por todos estos motivos, Periscope fue la tecnología pionera en el desarrollo del Social Media Live Streaming permitiendo que la opción del periodismo de emitir en directo evolucionase desde la decisión espontánea del redactor de sacar su móvil en el lugar de los hechos hasta ser parte indispensable de la estrategia de contenidos de los cibermedios.

Los temores de que el SMLS pudiese reemplazar a la televisión quedaron rápidamente desterrados por los motivos que su CEO y creador, Kayvon Beykpour, explicó en una entrevista a El País:

En el boxeo, el baloncesto, el béisbol, la televisión tiene ángulos, repeticiones, definición... Periscope sirve para hacer una idea de algo en primera persona. Puede mostrar la cara b, la parte de atrás de un gran evento, pero nunca competir. Los medios que se han sumado lo hacen para acercar a los presentadores, a las estrellas, para contar de otra manera. Es complementario (Beykpour, 2016).

Un ejemplo reciente es la emisión del 'Juicio del procés'1. La señal autorizada pertenece al Consejo General del Poder Judicial de España (Álvarez, 2019) y, ante el bajo interés de la televisiones generalistas de emitir las sesiones en directo, distintos cibermedios la han aprovechado para realizar emisiones SMLS. Concretamente en el caso de Periscope, destacan las publicaciones en directo de El País, 24 Horas, ABC, La Vanguardia, EFE, Cadena SER y El Mundo.

\subsubsection{Facebook Live}

Al constatar el éxito del SMLS en Periscope, Facebook comienza a implementar el live streaming a través de una funcionalidad dentro su app Mentions, dedicada al contenido exclusivo de celebridades. Así, desde la actualización del 10 de septiembre de 2015 es posible realizar emisiones en directo con esta red social (Lavrusik, 2015).

1. La Causa Especial 20907/2017 del Tribunal Supremo de España corresponde al juicio a líderes del proceso independentista catalán. 
Meses más tarde nació Facebook Live como una funcionalidad para los perfiles de usuarios y páginas de fans. Desde abril de 2017, todo usuario que se conecte con un dispositivo con cámara puede emitir en directo (Facebook for Media, 2017).

En ese mismo período, Fidji Simo², que en ese entonces era la jefa de vídeo de la compañía, anunció que Facebook Live era la red de SMLS con mayor número de reproducciones debido a que uno de cada cinco vídeos reproducidos en la red social correspondían a emisiones en directo (Constine, 2017).

Diversos editores periodísticos declararon cambiar Periscope por Facebook Live por los siguientes motivos: crecimiento estable del alcance orgánico, información más detallada de quién pudo visualizar el vídeo, seguimiento de hashtags, y mayores oportunidades de llegar a una audiencia masiva (López Pinares, 2017).

A ello se suma un segundo factor: el pago de 50 millones de dólares que realizó Facebook en 2016 a editores, marcas e influencers de todo el mundo para que emitiesen en directo en su plataforma. Los principales cibermedios beneficiados fueron BuzzFeed con 3,1 millones de dólares, The Washington Post con 3 millones de dólares, CNN con 2,5 millones de dólares y Huffington Post con 1,6 millones de dólares (Perlberg \& Sheetharaman, 2016).

Sin embargo, este último punto produjo una situación antinatural que trajo como consecuencia que, una vez terminado el subsidio, los cibermedios beneficiados disminuyeran su uso en un 94\% entre mayo de 2016 y diciembre de 2017 (Brown, 2018); que medios como The New York Times, en su condición de subsidiado, reconociesen que dudaban de su efectividad (Maheshwari \& Ember, 2018); y que otros, como Digiday, lo calificasen directamente como "un fracaso" (Patel, 2017).

La solución de la red social fue realizar un cambio de algoritmo a principios de 2018 que, entre otras variables, estimuló el alcance de las emisiones en directo (Mosseri, 2018) y trajo como consecuencia mejoras en los resultados de Facebook Live a medios de comunicación que no fueron subsidiados (Peterson, 2018a).

Pese a todos los escándalos de filtración de datos, Facebook continúa siendo la red social más utilizada en el mundo (StatCounter, 2019) y, por ello, diversos editores continúan confiando en esta para las emisiones en directo. Sin embargo, aún tiene un importante desafío por delante: ser capaz de frenar o de dar de baja con mayor rapidez contenidos violentos, especialmente asesinatos, en directo.

En este sentido, el primer caso se produjo en 2017, cuando Steve Stephens emitió un vídeo en directo desde Pensilvania, Estados Unidos, de 57 segundos, anunciando que mataría al primer vagabundo que se encontrase y, tras cumplir su cometido, su publicación estuvo en línea por más de dos horas antes de que Facebook eliminase el contenido (eldiario.es, 2017).

2. El viernes 15 de marzo de 2019, Fidji Simo fue nombrada directora general de Facebook; es decir, que es la máxima responsable de la red social. No confundir con la matriz empresarial Facebook Inc., cuyo director general continúa siendo Mark Zuckerberg. 
Como respuesta, la red social anunció en 2018 la creación de un chip inteligente para impedir este tipo de emisiones en directo (Clases de Periodismo, 2018). Sin embargo, este sistema falló rotundamente durante el atentado contra dos mezquitas en Christchurch, Nueva Zelanda, el 15 de marzo de 2019, debido a que el autor pudo emitir sus acciones por 17 minutos porque Facebook solo reaccionó al recibir un llamado de la policía local exigiendo la eliminación del contenido (Sánchez, 2019).

Todo esto ha generado un intenso debate sobre la capacidad que tienen las redes sociales para controlar este tipo de publicaciones porque, hasta ahora, la mayoría dependen de las denuncias de los usuarios. Por si fuera poco, tras el atentado que acabó con la vida de 50 personas, existe una mayor preocupación aún sobre cómo evitar que se repitan este tipo de publicaciones (ABC, 2019). Se ha propuesto realizar un diferecto, emisión con tiempo de retraso para verificar el contenido, pero Facebook ha comunicado oficialmente que aplicar este sistema tendría un alto coste y no aportaría mayores soluciones a este problema (Rosen, 2019).

\subsubsection{YouTube Live}

Curiosamente, la red social de vídeos de Google fue pionera en la emisión en directo: en 2008, realizó una serie de eventos simultáneos llamados YouTube Live para premiar a los primeros influencers que tuvo la plataforma (Dobuzinskis, 2008). Sin embargo, tuvo que pasar una década para que fuese posible que todo usuario pudiese realizar una publicación SMLS (Ong, 2018).

Fue ante el crecimiento de Periscope/Twitter y de Facebook Live que YouTube decidió apostar por el vídeo en directo, aunque en pleno auge del Social Media Live Streaming claramente tuvo dudas hacia dónde ir. En 2016, estudió la opción de crear una red social SMLS cuyo nombre tentativo fue YouTube Connect y que competiría directamente con Periscope (Yeung, 2016).

Sin embargo, con el tiempo se decidió reforzar a YouTube Live, que desde 2013 se había convertido en una opción de live streaming exclusiva para cuentas verificadas (MacNN, 2013), hasta convertirla en una funcionalidad SMLS que, a su vez, cuenta con YouTube Live Events como una variante para profesionales que deseen realizar emisiones multicámaras.

Actualmente, YouTube Live es el único canal Social Media Live Streaming que exige a los emisores solicitar una habilitación previa a la primera publicación en directo, la cual demora en promedio 24 horas en concretarse. En este período, se revisa la información de cada perfil para verificar que cumpla con las normas de la comunidad (YouTube, 2018).

Lo cierto es que, ante la crisis de Facebook y el bajo alcance comparativo que ofrecen Periscope y Twitter, diversos editores decidieron cambiarse a YouTube Live. De este modo, a finales de 2018, los medios digitales que llevaban por lo menos un par de años emitiendo en directo a través de la plataforma de Google observaron un continuo crecimiento en los resultados. 
En concreto, se trata de los casos de Time, GTLive y The Young Turks Network, cuyos responsables explicaron a Digiday que desconocen los verdaderos motivos por los cuales han observado este crecimiento, porque estos medios no han realizado grandes cambios en sus estrategias de contenidos SMLS. Por su parte, aunque YouTube niega un cambio de algoritmo, la sospecha de la industria acerca de este inesperado crecimiento de audiencia orgánica es un cambio no oficial en el posicionamiento de emisiones en directo (Peterson, 2018b).

En todo caso, lo que la industria periodística comprende es que YouTube Live prioriza los contenidos de larga duración en formato 24/7, por lo cual es posible encontrar emisiones en directo que comenzaron hace más de un año; y que cuenta con Super Chat, una herramienta que estimula tanto la conversación en directo como los aportes económicos de la audiencia hacia el emisor del contenido (Parker, 2017).

\section{Diseño y componentes de la investigación}

Como se ha mencionado en la introducción, el objeto de estudio de este trabajo corresponde a los canales SMLS de medios de comunicación de Chile con ocasión de la cobertura informativa de la conmemoración del $30^{\circ}$ aniversario del Plebiscito Nacional de 1988.

En consecuencia, el objetivo principal consiste en intentar identificar el rendimiento de los canales Social Media Live Streaming a través de los medios de comunicación digitales chilenos.

Los objetivos derivados son los siguientes:

- Describir las coberturas informativas de los medios digitales chilenos a través de las emisiones SMLS.

- Estudiar los impactos y resultados de medios digitales que utilizan Periscope/ Twitter, Facebook Live y/o YouTube Live para las emisiones de vídeos en directo.

Para abordar los objetivos mencionados, se plantean las siguientes preguntas de investigación:

- ¿Cuán útiles son Periscope/Twitter, Facebook Live y/o YouTube Live en las visibilidad de contenidos informativos a través de las redes sociales?

- ¿Qué estrategias de contenidos han aplicado los medios digitales chilenos en sus publicaciones Social Media Live Streaming?

\subsection{Marco teórico}

En estricto rigor, las videollamadas están cercanas a cumplir un siglo de existencia (Silva, 2017). Sin embargo, el live streaming es un término que solo es conocido desde hace una década y que, según sostienen diversos autores, solo ha tomado real fuerza a través de su uso y aplicación en las redes sociales (Rugg \& Burroughs, 2016; Rein \& Venturini, 2018).

Este recurso ha entregado una serie de facilidades al emisor de contenidos informati- 
vos (esto es, en nuestro caso, a cibermedios). A quien, según los principios de la Teoría del Periodismo -también conocida como Periodística-, se le conoce como un operador semántico: "el periodista está obligado a manipular lingüísticamente una realidad bruta para conseguir elaborar un mensaje adecuado mediante una acertada codificación" (Gomis, 1991, p. 37).

Si este mensaje adecuado se transmite a través de formatos digitales, estos se denominan como cibermedios, es decir como "sitios web que cumplen en internet el papel de los medios de comunicación social" (Palacios \& Díaz Noci, 2009, p. 21). Por lo tanto, la especialidad del periodismo dedicada a la difusión de contenidos informativos a través del ciberespacio se conoce como ciberperiodismo (Díaz Noci \& Salaverría, 2003, p. 17).

Cuando Marshall McLuhan (1996:46) plantea que "ningún medio tiene sentido o existe a solas, sino solamente en interacción constante con otros medios" permite la construcción del concepto ecología de los medios. Esta teoría, ampliamente profundizada por diversos autores (Postman, 2000; Scolari, 2010), contextualiza la vital importancia de que cada medio informativo genere diferentes extensiones complementarias a su formato nativo en el escenario digital actual.

\subsection{Metodología}

Para proporcionar el mayor rigor posible a cada una de las partes del presente artículo se ha realizado una revisión sistematizada tanto de publicaciones académicas, a través de la búsqueda y configuración de alertas en bases de datos especializadas, como de la industria, a través de redes sociales agregadoras de contenido, siguiendo los principios de la también llamada 'sistematic review' (Booth, et al., 2016). Esta revisión aportó los fundamentos conceptuales que nos han ayudado al diseño del estudio de caso.

En este sentido, lo anterior ha permitido la elaboración de un estudio de caso (Coller, 2005; Yin, 2018; Flyvbjerg, 2013; Thomas, 2011), cuya aplicación se traduce en los resultados que se presentan en los apartados siguientes. En esta línea, es importante describir las principales características del estudio realizado:

Fecha de toma de datos: 5 de octubre de 2018

Suceso informativo: Conmemoración de los 30 años del Plebiscito Nacional de Chile de 1988

Medios estudiados: Teletrece, La Tercera, TVN 24 Horas, CNN Chile, Ahora Noticias

(Meganoticias) $)^{3}$, Cooperativa y Publimetro

Canal(es) SMLS: Facebook Live, Periscope/Twitter y YouTube Live

Total de emisiones: 31 ( 25 en Facebook Live, 4 en Periscope/Twitter y 2 en YouTube Live)

Fecha y hora de obtención de la muestra: 10 de octubre de 2018 a las 23:59 (hora chilena)

Rendimiento e interacciones estudiadas:

- Facebook Live: emisiones, reproducciones, compartidos, total comentarios y reacciones por medio.

-Periscope y Twitter: emisiones, espectadores totales, espectadores en directo, espectadores en repetición, tiempo de visualización, tiempo de visualización por espectador, e interacciones en Twitter por medio.

-YouTube Live: emisiones, visualizaciones, comentarios, me gusta y no me gusta.

3. Ahora Noticias fue el nombre de los servicios informativos del canal de televisión chileno Mega durante la fecha estudiada. Desde el 23 de julio de 2019, estos han recuperado su nombre fundacional: Meganoticias. Para evitar equívocos, en este trabajo se conservará el nombre utilizado durante la fecha del estudio de caso. 
Finalmente, es importante destacar la aplicación de análisis de contenidos (Neuendorf, 2002) y análisis comparativos (Walk, 1998) que permiten una interpretación de los resultados obtenidos, cruzando los datos y dando mayor rigurosidad a las argumentaciones que se expondrán tanto en la descripción de los resultados por canal, como en la discusión y en las conclusiones.

\section{Resultados}

El miércoles 5 de octubre de 1988 se celebró el Plebiscito Nacional de Chile, un referéndum que permitió la salida de Augusto Pinochet del poder y el regreso a democracia a partir de 1990. De acuerdo a los resultados oficiales, tras escrutar alrededor de 7,5 millones de votos la opción 'No' superó por un 55,99\% a la opción 'Sí' (44,01\%) que habría extendido el gobierno militar, por lo menos, ocho años más (Tribunal Calificador de Elecciones de Chile, 1988).

El viernes 5 de octubre de 2018, con motivo del trigésimo aniversario del acto que permitió el posterior retorno a la democracia en Chile, se realizaron diversas conmemoraciones de la actividad, que incluyeron desde ceremonias solemnes hasta conciertos. Los medios de comunicación no estuvieron ajenos a la actividad, especialmente aquellos que ya existían en ese período que pudieron reutilizar sus contenidos de archivo.

comScore, empresa especializada en el marketing digital y métricas de audiencias, publicó en 2018 el Chile Media Index, un ranking que analiza las acciones de los cibermedios del país en las diferentes redes sociales tal como se puede ver en la tabla 1.

Siguiendo los parámetros del ranking, se buscaron emisiones en directo en cada uno de los 10 medios mencionados durante el 5/10/2018, seleccionándose para el estudio de caso solo aquellos que tuviesen relación con el 30 aniversario del plebiscito. De ellos, TVN 24 Horas $^{4}\left(1^{\circ}\right)$, Ahora Noticias $\left(3^{\circ}\right)$, La Tercera $\left(4^{\circ}\right)$, Cooperativa $\left(5^{\circ}\right)$, CNN Chile $\left(8^{\circ}\right)$ y Publimetro Chile $\left(10^{\circ}\right)$ cumplieron con tales requisitos. A ellos, se incluyó Teletrece debido el mismo Facebook ha destacado como un caso de éxito mundial sus emisiones en directo a través de Facebook Live (Facebook, 2016).

\begin{tabular}{|c|c|c|c|c|c|}
\hline \multicolumn{2}{|c|}{$\begin{array}{l}\text { Fuente: Shareablee } \\
\text { Plataformas: Facebook, Twit- } \\
\text { ter e Instagram } \\
\text { Chile, Mayo } 2018\end{array}$} & $\begin{array}{l}\text { Acciones } \\
\text { Cross-Platform } \\
\text { (Shares, Likes, } \\
\text { Comments, etc.) }\end{array}$ & $\begin{array}{l}\text { Share de } \\
\text { Acciones } \\
\text { Cross- } \\
\text { Platform }\end{array}$ & $\begin{array}{l}\text { Acciones } \\
\text { Post Cross- } \\
\text { Platform }\end{array}$ & $\begin{array}{c}\text { Audiencia } \\
\text { (Cross-Platform) }\end{array}$ \\
\hline \multicolumn{2}{|c|}{ Category Totals } & 20.846 .690 & $100,0 \%$ & 6.232 & 61.298.197 \\
\hline \multicolumn{2}{|c|}{ Category Averages } & 744.524 & $3,6 \%$ & 223 & 2.189 .221 \\
\hline 1 & 24horas.cl (Chile) & 5.838 .582 & $28,0 \%$ & 589 & 6.667 .577 \\
\hline 2 & Radio Bio Bio (Chile) & 2.658 .999 & $12,8 \%$ & 229 & 5.402 .798 \\
\hline 3 & Ahora Noticias (Chile) & 2.250 .040 & $10,8 \%$ & 330 & 2.764 .297 \\
\hline 4 & La Tercera & 2.241 .787 & $10,8 \%$ & 137 & 5.247 .438 \\
\hline 5 & Radio Cooperativa (Chile) & 2.022 .655 & $9,7 \%$ & 136 & 4.444 .867 \\
\hline
\end{tabular}

4. El nombre exacto del medio es Canal 24 Horas, medio televisivo público con contenido 24/7. Sin embargo, a partir de aquí se mencionará como TVN 24 Horas, incluyendo a su matriz Televisión Nacional de Chile como prefijo, para evitar confusiones con la señal homónima perteneciente a Radiotelevisión Española. 


\begin{tabular}{l|l|c|c|c|c|}
$\begin{array}{l}\text { Fuente: Shareablee } \\
\text { Plataformas: Facebook, Twit- } \\
\text { ter e Instagram }\end{array}$ & $\begin{array}{c}\text { Acciones } \\
\text { Cross-Platform } \\
\text { (Shares, Likes, } \\
\text { Chile, Mayo 2018 }\end{array}$ & $\begin{array}{c}\text { Share de } \\
\text { Acciones } \\
\text { Cross- } \\
\text { Comments, etc.) }\end{array}$ & $\begin{array}{c}\text { Acciones } \\
\text { Post Cross- } \\
\text { Platform }\end{array}$ & $\begin{array}{c}\text { Audiencia } \\
\text { (Cross-Platform) }\end{array}$ \\
\hline 6 & MEGA (Chile) & 1.097 .643 & $5,3 \%$ & 232 & 3.648 .641 \\
\hline 7 & TVN (Chile) & 1.020 .488 & $4,9 \%$ & 340 & 5.005 .016 \\
\hline 8 & CNN Chile & 773.091 & $3,7 \%$ & 158 & 4.032 .823 \\
\hline 9 & CDF (Chile) & 574.770 & $2,8 \%$ & 146 & 905.988 \\
\hline 10 & Publimetro (Chile) & 573.375 & $2,8 \%$ & 67 & 1.366 .110 \\
\hline
\end{tabular}

Tabla 1: Resultados del Chile Media Index 2018 (DossierNet, 2018).

Tal como se mencionó en el apartado correspondiente al estudio de caso, se analizaron un total de 31 emisiones en 7 cibermedios a través de Facebook Live (tabla 2), Periscope/Twitter (tabla 3) y YouTube Live (tabla 4).

\begin{tabular}{|l|c|c|c|c|c|}
\multicolumn{1}{r}{ Medio } & $\begin{array}{c}\text { Número de } \\
\text { emisiones }\end{array}$ & $\begin{array}{c}\text { Total de } \\
\text { reproducciones }\end{array}$ & $\begin{array}{c}\text { Total de } \\
\text { compartidos }\end{array}$ & $\begin{array}{c}\text { Total de } \\
\text { comentarios }\end{array}$ & $\begin{array}{c}\text { Total de } \\
\text { reacciones }\end{array}$ \\
\hline Teletrece & 11 & 157.262 & 333 & 1.421 & 1.594 \\
\hline La Tercera & 2 & 12.377 & 20 & 171 & 168 \\
\hline TVN 24 Horas & 3 & 57.061 & 196 & 637 & 802 \\
\hline CNN Chile & 1 & 5.900 & 13 & 72 & 71 \\
\hline Ahora Noticias & 6 & 170.681 & 732 & 3.492 & 4.876 \\
\hline Cooperativa & 1 & 15.967 & 168 & 261 & 426 \\
\hline Publimetro Chile & 1 & 1.481 & 3 & 32 & 22 \\
\hline Promedio & 3,6 & 16.829 & 59 & 243 & 318 \\
\hline Acumulado & 25 & 420.729 & 1.465 & 6.086 & 7.959 \\
\hline
\end{tabular}

Tabla 2: Rendimiento e interacciones de medios chilenos en Facebook Live durante los 30 años del plebiscito. | Fuente: Elaboración propia.

\begin{tabular}{|c|c|c|c|c|c|c|c|}
\hline Medio & $\begin{array}{l}\text { Número } \\
\text { de emi- } \\
\text { siones }\end{array}$ & $\begin{array}{l}\text { Total de } \\
\text { espec- } \\
\text { tadores }\end{array}$ & $\begin{array}{l}\text { Total de } \\
\text { especta- } \\
\text { dores en } \\
\text { directo }\end{array}$ & $\begin{array}{l}\text { Total de } \\
\text { especta- } \\
\text { dores en } \\
\text { repetición }\end{array}$ & $\begin{array}{c}\text { Tiempo } \\
\text { total de } \\
\text { visualiza- } \\
\text { ción }\end{array}$ & $\begin{array}{l}\text { Tiempo de } \\
\text { visualiza- } \\
\text { ción por } \\
\text { espectador }\end{array}$ & $\begin{array}{l}\text { Total de } \\
\text { interac- } \\
\text { ciones en } \\
\text { Twitter }\end{array}$ \\
\hline La Tercera & 1 & 311 & 192 & 119 & $4: 25: 00$ & $0: 00: 51$ & 7 \\
\hline TVN 24 Horas & 3 & 4.088 & 1.847 & 2.241 & 114:15:00 & $0: 04: 22$ & 121 \\
\hline Promedio & 2 & 1.100 & 510 & 590 & 29:40:00 & 0:01:18 & 32 \\
\hline Acumulado & 4 & 4.399 & 2.039 & 2.360 & $118: 40: 00$ & 0:05:13 & 128 \\
\hline
\end{tabular}

Tabla 3: Rendimiento e interacciones de medios chilenos en Periscope y Twitter durante los 30 años del Plebiscito. | Fuente: elaboración propia.

\begin{tabular}{|c|c|c|c|c|c|}
\hline Medio & $\begin{array}{l}\text { Número de } \\
\text { emisiones }\end{array}$ & $\begin{array}{c}\text { Total de } \\
\text { visualizaciones }\end{array}$ & $\begin{array}{l}\text { Total de } \\
\text { comentarios }\end{array}$ & $\begin{array}{l}\text { Total de me } \\
\text { gusta }\end{array}$ & $\begin{array}{c}\text { Total de no } \\
\text { me gusta }\end{array}$ \\
\hline Teletrece & 1 & 21.650 & 8 & 245 & 31 \\
\hline La Tercera & 1 & 387 & 0 & 1 & 2 \\
\hline Promedio & 1 & 11.019 & 4 & 123 & 17 \\
\hline Acumulado & 2 & 22.037 & 8 & 246 & 33 \\
\hline
\end{tabular}

Tabla 4: Rendimiento e interacciones de medios chilenos en YouTube Live durante los 30 años del Plebiscito. | Fuente: Elaboración propia. 
A continuación, se presenta un análisis por de las estrategias de contenidos SMLS realizadas por cada cibermedio estudiado.

\subsection{Teletrece}

La señal informativa de Canal 13 realizó un total de 12 emisiones sobre el plebiscito: 11 a través de Facebook Live y una a través de YouTube Live, esta última duró un total de 20 horas 41 minutos y 55 segundos, aunque luego publicó en su canal de YouTube una versión más reducida de casi 4 horas de duración.

En cuanto a contenidos, Teletrece realizó un especial informativo reviviendo a través de un minisitio (microsite $)^{5}$ y de sus redes sociales el minuto a minuto del plebiscito como si estuviese emitiendo en directo. Así, mientras en las señales web y YouTube se realizaba una emisión sin cortes, a través de Facebook Live se priorizaban los momentos destacados de la jornada, tales como la entrega de cómputos y las históricas declaraciones del comandante en jefe de la Fuerza Área de la época, Fernando Matthei, que reconocía la derrota electoral minutos antes de la entrega de los resultados oficiales ${ }^{6}$.

El único contenido de Teletrece alusivo a este tema que se emitió en estricto directo fue el acto conmemorativo realizado por el gobierno de Sebastián Piñera desde el Palacio de La Moneda con una duración superior a los 25 minutos a través de Facebook Live. Es importante destacar que en todo el especial informativo se usó el hashtag \#Plebiscito88.

\subsection{La Tercera}

De todos los medios estudiados, el periódico generalista fue el único que realizó emisiones en todos los canales SMLS estudiados. Sus 4 publicaciones SMLS (dos en Facebook Live, una en Periscope/Twitter y una en YouTube Live) se extraen dos tipos de contenidos principales: el ya citado evento en La Moneda y una tertulia en estudios con académicos y redactores del periódico.

Al igual con Teletrece, La Tercera creó un microsite en el que publicó informaciones minuto a minuto del plebiscito 7 . También es importante mencionar que en sus emisiones en directo se empleó su hashtag habitual para el SMLS: \#LTenVivo.

\subsection{TVN 24 Horas}

La señal informativa de la televisión pública chilena publicó 6 emisiones en directo (3 en Facebook Live y 3 en Periscope/Twitter), las cuales corresponden a tres contenidos principales emitidos en simulcasting: un reportaje con entrevistas a los vocales de mesas electorales del plebiscito, la reemisión de la última franja electoral televisiva de las campañas por cada opción, y la reemisión de la última entrega de resultados.

\section{5. http://www.t13.cl/plebiscito88}

6. https://www.facebook.com/teletrece/videos/1852863038084791/

\section{7. http://plebiscito.latercera.com/}

8. Se considera como simulcasting las emisiones con idéntico contenido que se emiten en más de un canal SMLS, pese a que ni la duración ni el horario de emisión coincidan de manera exacta. 
Coincidentemente TVN 24 Horas, al igual que su competidor Teletrece, utilizó el hashtag \#Plebiscito88.

\subsection{Ahora Noticias}

Medio informativo perteneciente a Mega, el canal de televisión en abierto más visto en Chile durante 2018 (Ramírez, 2019), que realizó seis publicaciones sobre el tema en la jornada, todas ellas a través de Facebook Live. Sus contenidos fueron todos en estricto directo y correspondían tanto al acto gubernamental como al evento organizado por políticos de oposición y exintegrantes del comando del "No" en el Paseo Bulnes de la ciudad de Santiago.

Este evento contó con la presencia de diversos grupos musicales, y gracias a su emisión, Ahora Noticias obtuvo los mejores resultados durante la jornada. De hecho, el vídeo más visto corresponde a la primera parte del evento se emitió a las 18:12 (hora local) del día señalado, tuvo una duración de 2 horas y 10 minutos, y obtuvo 52.548 reproducciones, 1.100 comentarios, 245 compartidos y 1.200 reacciones (me gusta, me encanta, me divierte, me asombra, me entristece y me enoja) ${ }^{9}$.

Es importante destacar, además, que en las publicaciones SMLS de Ahora Noticias se priorizó el contenido en formato vertical, pensando en el tráfico móvil y en que en sus publicaciones no hay presencia de hashtags, aunque sí de emoticonos.

\subsection{CNN Chile, Cooperativa y Publimetro Chile}

Cada uno de los medios restantes solo realizó una publicación en directo sobre el tema a través de Facebook Live. CNN Chile, la señal informativa del Grupo Turner Chile, emitió una entrevista desde plató a Genaro Arriagada, exsecretario ejecutivo del comando del 'No'; y Publimetro Chile, edición local del periódico gratuito de Metro International, también se sumó a la emisión en directo del acto realizado en el palacio de gobierno.

Párrafo aparte merece la emisión de Cooperativa, emisora radial con un alto compromiso con los derechos humanos durante el gobierno de Augusto Pinochet (Rivera Araneda, 2008, pp. 80-81). Por ello, realizaron un reportaje de poco más de 90 minutos con un resumen de los principales hitos que informaron en aquella jornada, los audios fueron apoyados con imágenes de los hechos más destacados y un reloj que marcaba la hora del día en que se emitió el contenido original ${ }^{10}$.

\section{Discusión}

Para saber en qué canal SMLS han tenido mejores resultados los cibermedios chilenos estudiados conviene realizar una estimación de cuántos usuarios alcanzaron por segundo emitido. Para ello, se realiza en cada caso una división del número total de reproducciones por el número total de segundos emitidos como se puede ver a continuación: 
- Facebook Live: 5,65 usuarios por segundo emitido

- Periscope/Twitter: 0,9 usuarios por segundo emitido

- YouTube Live: 0,76 usuarios por segundo emitido

De este modo se aplica la premisa de a mayor número de emisiones, mayores oportunidades de visualizaciones. Aun así, no está de más recordar las diferencias en las estadísticas públicas que entrega cada canal SMLS. Periscope, por ejemplo, no diferencia qué reproducciones provienen de su app o cuáles desde Twitter, pero sí especifica cuáles fueron en el directo y cuáles en la repetición. Mientras que Facebook y YouTube no hacen diferenciación alguna.

Siguiendo con la plataforma de vídeos de Google es importante tener en cuenta que las dos emisiones de YouTube Live estudiadas comenzaron el 5 de octubre de 2018 y que, gracias a que fueron editadas para su publicación en el canal luego de finalizado el directo, ha sido posible su inclusión en este estudio.

Otro aspecto que permite establecer comparativas de resultados según el canal social de cada medio específico es la cobertura a un suceso informativo en común. Como ya se ha visto en el apartado anterior, de las 31 emisiones estudiadas 4 corresponden al acto conmemorativo realizado por el presidente Sebastián Piñera en el Palacio de La Moneda, todas ellas fueron realizadas a través de Facebook Live con los siguientes resultados por cada medio:

1) Teletrece: 11.631 reproducciones

2) Ahora Noticias: 11.236 reproducciones

3) La Tercera: 10.699 reproducciones

4) Publimetro: 1.481 reproducciones

Como se puede apreciar, el medio con más reproducciones acumuladas (Ahora Noticias) no es el más visto para este suceso, aunque la diferencia entre los tres primeros es muy pequeña, pues es menor a las mil reproducciones.

También es importante mencionar que se detecta a través del estudio de caso un mayor volumen de resultados de medios de comunicación con señal televisiva como formato nativo. Un factor que puede influir es su mayor capacidad técnica para realizar variaciones en las emisiones, tal como sucedió con la adaptación del contenido vertical realizada por Ahora Noticias.

Existen una serie de artículos académicos que reafirman esta premisa (Mancebo García, 2016; Hutchins \& Sanderson, 2017; Herrero de la Fuente, 2018; Herrero de la Fuente \& García Domínguez, 2019): las oportunidades que entrega el SMLS para la televisión de complementar su experiencia, y no reemplazarla como ya explicaba el CEO de Periscope, Kayvon Beykpour.

Finalmente, se destaca la presencia de elementos interactivos con la audiencia. Este fue el caso de TVN 24 Horas que, en sus emisiones a través de Facebook Live, preguntó a sus espectadores por qué opción votaron en 1988. 


\section{Conclusiones}

Este trabajo permite aportar resultados que clarifican diversas dimensiones del objeto de estudio. Por un lado, hemos procedido a una caracterización del SMLS en términos eminentemente periodísticos, guiados no solo por nuestros intereses teóricos, sino también por el convencimiento de que la sostenibilidad de los medios pasa por entender estos nuevos formatos y sus posibilidades de engagement.

Además, se presenta el diseño de un estudio de caso en diferentes canales de emisiones de video en directo a través de las redes sociales que se puede aplicar a otras investigaciones, y finalmente, este estudio también se aplica a la cobertura de un suceso relevante por su importancia histórica a través del análisis de diferentes medios de comunicación de Chile.

En sendos apartados de este trabajo, y apoyados en tablas con datos detallados, se muestran las coberturas informativas de los medios analizados para la ocasión del evento informativo. Aunque en los apartados correspondientes se ofrece información detallada que, a su vez, ha sido sintetizada en tablas, es posible destacar como rasgo más característico de las coberturas analizadas que Facebook Live es el canal SMLS más usado y que, al mismo tiempo, brinda mejores resultados como se ha podido comprobar también en otros trabajos (Cruz, 2018).

Así mismo, hemos entregado un contexto general del SMLS y su vínculo con el periodismo. Además, el estudio de caso ha permitido mostrar los datos de impacto detallados, utilizando los diversos datos recogidos y aplicando también por nuestra parte estrategias de síntesis que ayudasen a interpretarlos. En este sentido, creemos que algunos de los impactos más significativos se pueden expresar en los siguientes resultados: el medio digital con mayor reproducciones acumuladas es Ahora Noticias (170.681), el medio con mayor número de emisiones es Teletrece (12) y los contenidos más vistos corresponden al concierto de celebración realizado en el Paseo Bulnes, emitido por Ahora Noticias, y el recuerdo de las declaraciones del general Matthei reconociendo la derrota electoral, emitido por Teletrece.

Como se ha analizado ampliamente, los canales SMLS -independiente de su naturaleza- se han convertido en un complemento (y en ningún caso un reemplazo) a las experiencias informativas que cada medio de comunicación ofrece a sus audiencias. El estudio de caso pone de manifiesto que, gracias a la exploración de cada canal, los cibermedios que realizan emisiones en directo a través de las redes sociales ya saben qué canal(es) son más adecuados para la visibilidad de sus contenidos audiovisuales.

Además, con la toma de datos del estudio ha sido posible descubrir que las estrategias seguidas por los medios han consistido principalmente en utilizar los diferentes canales SMLS del siguiente modo: Facebook Live como el medio principal para las emisiones, YouTube Live como un medio para emisiones de larga duración, y Periscope/Twitter como un medio de refuerzo en el que se reemiten contenidos que también están presentes en Facebook y/o YouTube.

Por último, cerrar este trabajo poniendo especial énfasis a las principales aportaciones 
hacia nuestro ámbito de estudio. En primer lugar, se presentó una caracterización del SMLS en términos de interés periodístico. En segundo lugar, se proporcionó el diseño de un estudio de caso que puede extrapolarse a otros trabajos y que, por tanto, puede ser útil a otros equipos de investigación. Por último, los resultados detallados del estudio de caso aportan datos concretos que dan mayor rigurosidad a las consideraciones teóricas sobre esta clase de canales $y$, también, proveen datos y análisis que pueden ser útiles a estudiosos de los cibermedios y a profesionales del área de innovación de medios digitales.

\section{Referencias}

$A B C, 2019$. Las redes sociales no pueden controlar el contenido violento que se emite en directo. [En línea] Available at: https://www.abc.es/tecnologia/redes/abci-redessociales-no-pueden-controlar-contenido-violento-emite-directo-201903151042 video.html

Aguayo, D., 2015. Periscope: ¿una competencia real para los medios?. [En línea] Available at: http://vergara240.udp.cl/periscope-una-competencia-real-para-losmedios/

Álvarez, M., 2019. El juicio del caso "procés" se transmitirá en "streaming", en directo, a través del portal del CGPJ. [En línea] Available at: https://confilegal.com/20190201-eljuicio-del-caso-proces-se-transmitira-en-streaming-en-directo-a-traves-del-portaldel-cgpj/

Apablaza-Campos, A. \& Codina, L., 2018b. Social Media Live Streaming: estudio de caso y diseño de matriz de análisis. Cuadernos.info, Issue 40, pp. 161-180.

Apablaza-Campos, A., Codina, L. \& Pedraza-Jiménez, R., 2018a. Newsonomics in the Interactive Era: Dimensions of Sustainability in the News Media. En: M. Pérez-Montoro, ed. Interaction in Digital News Media. London: Palgrave MacMillan, pp. 115-146.

Argila, J., 2017. How to create video news that rocks in social media, Oxford: Reuters Institute.

Beykpour, K., 2016. "Periscope muestra la cara b de un evento, no compite con los medios" [Entrevista] (17 mayo 2016).

Booth, A., Sutton, A. \& Papaioannou, D., 2016. Systematic Approaches to a Successful Literature Review. Londres: Sage.

Brown, P., 2018. RIP Facebook Live: As subsidies end, so does publisher participation. [En línea] Available at: https://www.cjr.org/tow_center/facebook-live-over-as-paymentsend.php

Clases de Periodismo, 2018. Facebook filtrará los videos en directo con un chip inteligente. [En línea] Available at: http://www.clasesdeperiodismo.com/2018/05/30/facebookfiltrara-los-videos-en-directo-con-un-chip-inteligente/

Coller, X., 2005. Estudio de casos. Volumen 30 de Cuadernos metodológicos. Madrid: Centro de Investigaciones Sociológicas.

Constine, J., 2017. One in five Facebook videos is Live as it seizes the verb. [Online] Available at: https://techcrunch.com/2017/04/06/live-video/

Cruz, J., 2018. Voting with Views: The Role of Facebook Live in Puerto Rico's Gubernatorial Election. En: A. V. Laskin, ed. Social, mobile, and emerging media around the world: communication case studies. Lanham(Maryland): Lexington Books, pp. 25-40. 
Díaz Noci, J. \& Salaverría, R., 2003. Manual de redacción ciberperiodística. Barcelona: Ariel.

Dobuzinskis, A., 2008. YouTube ventures into live event webcasting. [En línea] Available at: https://www.reuters.com/article/us-media-youtube-idUSTRE4AB40U20081112

DossierNet, 2018. ComScore Presenta "Chile Media Index 2018". [En línea] Available at: $\quad$ https://www.dossiernet.com.ar/articulo/comscore-presenta-chile-mediaindex-2018-/16252

Dotan, T., 2017. Twitter Broadens Live Video Push. [En línea] Available at: https://www. theinformation.com/articles/twitter-broadens-live-video-push

Eadicicco, L., 2015. Apple's 25 Best iPhone Apps of the Year. [En línea] Available at: http:// time.com/4143145/best-iphone-apps-2015/

eldiario.es, 2017. EEUU busca a un hombre que retransmitió un asesinato en Facebook. [En línea] Available at: https://www.eldiario.es/tecnologia/EEUU-retransmitio-asesinatoFacebook-Live_0_633787014.html

Facebook for Media, 2017. Live Updates for Publishers. [En línea] Available at: https:// www.facebook.com/facebookmedia/blog/live-updates-for-publishers

Facebook, 2016. Editores de noticias de todo el mundo triunfan con Facebook Live. [En línea] Available at: https://www.facebook.com/facebookmedia/success-stories/ globalnews-live\#chile

Flyvbjerg, B., 2013. Case Study. En: N. K. Denzin \& Y. S. Lincoln, edits. Strategies of Qualitative Inquiry. Londres: SAGE Publications, pp. 169-204.

Fundación Gabriel García Márquez para el Nuevo Periodismo Iberoamericano, 2016. Ocho claves para entender las audiencias digitales en Colombia, Bogotá: FNPI.

Gomis, L., 1991. Teoría del periodismo: cómo se forma el presente. Barcelona: Paidós.

Hansen, E. \& Goligoski, E., 2018. Guide to Audience Revenue and Engagement, New York: Tow Center for Digital Journalism.

Herrero de la Fuente, M., 2018. Facebook y la televisión en directo: el uso de Facebook Live en LaSexta. En: Nuevas realidades en la comunicación audiovisual. Madrid: Tecnos, pp. 221-236.

Herrero de la Fuente, M. \& García Domínguez, A., 2019. Facebook Live y la televisión social: el uso del streaming en Antena 3 y laSexta. VivatAcademia, Issue 146, pp. 43-70.

Hutchins, B. \& Sanderson, J., 2017. The primacy of sports television: Olympic media, social networking services, and multi-screen viewing during the Rio 2016 games. Media International Australia, 164(1), pp. 32-43.

Ingram, M., 2016. Facebook May be Playing Catch-Up on Video, but It Is Going All In. [Online] Available at: http://fortune.com/2016/03/02/facebook-video/

Instagram, 2019. ¿Cómo inicio un vídeo en directo en Instagram?. [En línea] Available at: https://help.instagram.com/292478487812558?helpref=faq_content [Último acceso: 26 febrero 2019].

Kalogeropoulos, A., Cherubini, F. \& Newman, N., 2016. Digital News Proyect: The future of online news video, Oxford: Reuters Institute.

Kemp, S., 2019. Digital 2019, Londres: Hootsuite.

Lavrusik, V., 2015. New: Facebook Mentions and Live for Verified Profiles. [En línea] Available at: https://media.fb.com/2015/09/10/mentions-and-live-for-verified-profiles/ 
Levis, P., 2015. The Baltimore riots: the night on Periscope - video. [Online] Available at: https://www.theguardian.com/us-news/video/2015/apr/28/thebaltimore-riots-the-night-on-periscope-video

López Pinares, C., 2017. Cómo usar Facebook Live en el periodismo para mayor interacción con los usuarios: lecciones de medios en español. [En línea] Available at: https://knightcenter.utexas.edu/es/blog/00-17964-como-usar-facebook-live-en-elperiodismo-para-mayor-interaccion-con-los-usuarios-lecci

Lunden, I., 2019. LinkedIn debuts LinkedIn Live, a new live video broadcast service. [En línea] Available at: https://techcrunch.com/2019/02/11/linkedin-debuts-linkedin-livea-new-live-video-broadcast-service/

MacNN, 2013. YouTube opens live streaming for all verified accounts. [En línea] Available at: $\quad$ http://www.macnn.com/articles/13/12/13/youtube.abandons.100.subscriber. limit.for.live.streaming/

Maheshwari, S. \& Ember, S., 2018. The End of the Social News Era? Journalists Brace for Facebook's Big Change. [Online] Available at: https://www.nytimes.com/2018/01/11/ business/media/facebook-news-feed-media.html

Mancebo García, M., 2016. Innovación en formatos audiovisuales. El uso de herramientas de live streaming en Antena 3 y Univision. Miguel Hernández Communication Journal, Issue 7, pp. 325-349.

McLuhan, M., 1996. Comprender los medios de comunicación. Las extensiones del sur humano. Barcelona: Paidós.

Morales Vargas, A., 2018. Plataforma ciudadana y streaming en redes sociales: usuarios en directo (Seminario DigiDoc) [Citizen platform and streaming on social networks: live users [DigiDoc Seminar]]. [Online] Available at: https://observatoriocibermedios.upf. edu/3102-2

Mosseri, A., 2018. Bringing People Closer Together. [En línea] Available at: https:// newsroom.fb.com/news/2018/01/news-feed-fyi-bringing-people-closer-together/

Neuendorf, K. A., 2002. The Content Analysis Guidebook. Thousand Oaks: Sage.

Ong, T., 2018. YouTube live-streaming gets easier from computers and phones. [En línea] Available at: https://www.theverge.com/2018/3/21/17146288/youtube-livestreaming-tool-chrome-browsers-camera-app-android

Palacios, M. \& Díaz Noci, J. edits., 2009. Online journalism : Research methods. A multidisciplinary approach in comparative perspective. Salvador de Bahía: Argitalpen Zerbitzua.

Panzarino, M. \& Tsotsis, A., 2015. Twitter Buys Live Streaming App Periscope. [En línea] Available at: https://techcrunch.com/2015/03/04/twitter-in-talks-with-live-streamingapp-periscope/

Parker, L., 2017. A Chat With a Live Streamer Is Yours, for a Price. [En línea] Available at: https://www.nytimes.com/2017/04/12/technology/personaltech/paying-for-livestream-chat.html

Patel, S., 2017. Facebook plans to stop paying publishers to make news feed videos. [Online] Available at: https://digiday.com/media/facebook-plans-to-stop-paying-publishersto-make-news-feed-videos/ 
Periscope, 2017. Quiénes somos. Nuestra historia. [En línea] Available at: https://www. periscope.tv/about [Último acceso: 17 marzo 2019].

Perlberg, S. \& Sheetharaman, D., 2016. Facebook Signs Deals With Media Companies, Celebrities for Facebook Live. [En línea] Available at: https://www.wsj.com/ articles/facebook-signs-deals-with-media-companies-celebrities-for-facebooklive-1466533472

Peterson, T., 2018a. Despite subsidies disappearing, some publishers see hope for Facebook Live post-algorithm change. [Online] Available at: https://digiday.com/media/despitesubsidies-disappearing-publishers-see-hope-facebook-live-post-algorithm-change/

Peterson, T., 2018b. YouTube channels are seeing a lift in live video viewership. [En línea] Available at: https://digiday.com/media/youtube-channels-seeing-lift-live-videoviewership/?utm source=twitter\&utm medium=social\&utm campaign=digidaydis

Pichihua, S., 2015. Así usó un periodista Periscope para la cobertura sobre los refugiados. [En línea] Available at: http://www.clasesdeperiodismo.com/2015/09/14/asi-uso-unperiodista-periscope-para-la-cobertura-sobre-los-refugiados/

Postman, N., 2000. The Humanism of Media Ecology. Nueva York, Fordham University, pp. 10-16.

Ramírez, C., 2019. Cada chileno consumió, en promedio, 3,31 horas diarias de televisión en 2018, y la TV abierta sigue liderando la sintonía. [En línea] Available at: http://www. economiaynegocios.cl/noticias/noticias.asp?id=556311

Rein, K. \& Venturini, T., 2018. Ploughing digital landscapes: How Facebook influences the evolution of live video streaming. New Media \& Society, 20(9), pp. 3359-3380.

Rivera Araneda, C. A., 2008. La verdad está en los hechos: una tensión entre objetividad y oposición. Radio Cooperativa en dictadura. Historia, 41(1), pp. 79-98.

Rosen, G., 2019. A Further Update on New Zealand Terrorist Attack. [En línea] Available at: https://newsroom.fb.com/news/2019/03/technical-update-on-new-zealand/

Rugg, A. \& Burroughs, B., 2016. Periscope, live-streaming and mobile video culture. In: R. Lobato \& J. Meese, eds. Geoblocking and global video culture. s.I.:Institute of Network Cultures, pp. 64-73.

Sánchez, J., 2019. Polémica alrededor de Facebook Live: un tirador de Nueva Zelanda emitió 17 minutos de masacre. [En línea] Available at: https://www.abc.es/tecnologia/redes/ abci-polemica-alrededor-facebook-live-tirador-nueva-zelanda-emitio-17-minutosmasacre-201903150904 noticia.html

Scolari, C., 2010. Ecología de los medios. Mapa de un nicho teórico. Quaderns del CAC, XIII(1), pp. 17-25.

Silva, M., 2017. Hoy se cumplen 90 años de la primera videollamada de la historia. [En línea] Available at: https://www.fayerwayer.com/2017/04/hoy-se-cumplen-90-anosde-la-primera-videollamada-de-la-historia/

SimilarWeb, 2019. Top Websites Ranking. [En línea] Available at: https://www.similarweb. com/top-websites

Southern, L., 2018. With Facebook Live views falling, BuzzFeed looks to Twitch. [En línea] Available at: https://digiday.com/media/facebook-live-views-falling-buzzfeed-lookstwitch/?utm campaign=digidaydis\&utm medium=social\&utm source=twitter 
StatCounter, 2019. Social Media Stats Worldwide July 2017 - Oct 2018 Edit Chart Data. [En línea] Available at: http://gs.statcounter.com/social-media-stats\#monthly-201707201810-bar [Último acceso: 24 febrero 2019].

Thomas, G., 2011. How to do your Case Study: A Guide For Students And Researchers. $1^{\text {a }}$ edición ed. Londres: SAGE Publications.

Tribunal Calificador de Elecciones de Chile, 1988. Sentencia Plebiscito 1988, Santiago de Chile: s.n.

Walk, K., 1998. How to Write a Comparative Analysis. [En línea] Available at: https://writingcenter.fas.harvard.edu/pages/how-write-comparative-analysis [Último acceso: 2 marzo 2019].

Yeung, K., 2016. Google is building YouTube Connect, a livestreaming app to take on Periscope. [En línea] Available at: https://venturebeat.com/2016/03/23/google-isbuilding-youtube-connect-a-live-streaming-app-to-take-on-periscope/

Yin, R. K., 2018. Case Study Research and Applications: Design and Methods. Sixth ed. Los Angeles: SAGE Publications.

YouTube, 2018. YouTube Live. [En línea] Available at: https://www.youtube.com/live dashboard_splash [Último acceso: 25 marzo 2019].

\section{Agradecimientos}

Esta investigación cuenta con el apoyo de CONICYT (Comisión Nacional Científica y Tecnológica de Chile), a través del programa "Becas Chile" Doctorado en el Extranjero, número de folio 72170334 correspondiente a Alexis Apablaza-Campos y número de folio 72180115 correspondiente a Alejandro Morales Vargas.

Asimismo, esta investigación forma parte del proyecto "Creación y contenido interactivo en la comunicación de información audiovisual: audiencias, diseño, sistemas y formatos". Ref: CSO2015-64955-C4-2-R (MINECO/FEDER), Ministerio de Economía y Competitividad (España). 